\title{
External Pop-out Cesarean Section: A Novel Technique for Supporting the Lower Uterine Segment During Fetal Head Extraction
}

\section{Original Article}

\author{
Ali El Saman', Ahmed M. Abbas ${ }^{1}$, Mohammed K. Ali', Essam R. Othman', \\ Ibrahim I. Mohamed ${ }^{1}$, Mohamed Khalaf ${ }^{1}$, Mustafa Bahloul', Dina A. El Saman ${ }^{2}$ \\ ${ }^{1}$ Department of Obstetrics and Gynecology, Woman's Health Hospital, Assiut University, \\ Assiut, Egypt, ${ }^{2}$ Faculty of Medicine, Assiut University, Assiut, Egypt
}

\begin{abstract}
Objectives: To assess the safety and feasibility of a novel technique, External Pop-out (EPO), for supporting the lower uterine segment during fetal head extraction in cesarean section (CS).

Study design: Prospective cohort study.

Patients and Methods: The current research is a registered (NCT02755168) study compassing the new technique, EPO, for head extraction during CS. The technique was performed on single, living, cephalic, elective cesarean deliveries at Women's Health Hospital, Assiut University, Egypt. The primary study outcomes were; the feasibility and the rate of incision extensions with the External Pop-out technique. The secondary outcomes included the easiness score and the learning curve of this technique on five senior obstetricians' trainee.

Results: The EPO technique was successful in 845/877 cases (96.3\%) but impossible in the remaining 32 case because of extensive pelvic adhesions. The rate of occurrence of minor extensions associated with EPO was $8 / 845(0.95 \%)$ with no major extensions in succeeded cases. There was a statistically significant difference between the mean of easiness score in the early 5 cases and the next 5 cases in 4 out of 5 trainees. The remaining trainee reported a higher easiness score earlier from his $4^{\text {th }}$ case. The mean of total scores in the late cases was also significantly higher than the early cases $(p=0.0001)$.

Conclusions: External Pop-out technique is feasible with low rates of uterine incisions extensions. Moreover; this technique is easily learned and performed by obstetricians.
\end{abstract}

Submitted: 1 September 2016, Accepted: 1 February 2017, Published: $\mathrm{x}-\mathrm{X}-\mathrm{X}$

Key Words: Cesarean Section; Fetal Head Extraction; Maternal Morbidity; Uterine Extension.

Corresponding Author: Dr. Ahmed M. Abbas, MD, Department of Obstetrics and Gynecology, Assiut University, Egypt, Woman's Health Hospital, 71511, Assiut Egypt., Tel.: 2088 2414616., E-mail: bmr90@hotmail.com

ISSN: 2090-7265, February 2017, Vol. 7, No. 1

\section{INTRODUCTION}

Caesarean section (CS) is the delivery of the fetus through a surgical incision in the abdominal wall (laparotomy) and uterine wall (hysterotomy) ${ }^{1}$. In the last decades, the rate of cesarean delivery has increased progressively until becomes the most common operation performed all over the world ${ }^{2}$.

A multitude of efforts had been done aiming at reducing CS related maternal morbidities; most of them are related to technical modifications of how to open and how to close the abdominal and uterine incisions ${ }^{3}$. The comparative studies of blunt versus sharp extension of the uterine incision showed a reduction of the incidence of unintended extension from $8.8 \%$ to $4.8 \%{ }^{4}$.

The vulnerability of the lower uterine segment (LUS) for tears is related to the stage of labor. The frequency of unintended extension was reported to be $15.5 \%$, and $35.0 \%$ in cases operated in first and second stages of labor, respectively ${ }^{5}$.

As with any surgical operation, anticipating difficulties during cesarean delivery and avoiding these difficulties is always the greatest practice. Although much has been written about techniques for managing difficult head extraction during vaginal deliveries, the reports addressed the management of difficult head delivery during CS in literature are scarce ${ }^{6}$.

The original techniques of fetal head extraction entail the introduction of the obstetricians' hands or other instruments into the LUS. This puts the LUS at risk of damage and incision extensions with its consequences of increased blood loss, increased operative time, 
infection adhesions and blood transfusion. Adherence to the available the generated good quality evidence bases practice in CS is anticipated to decrease such morbidities?

The idea of the present technique was derived from the fact that during vaginal delivery the main task of the obstetrician is to support the perineum while the fetal head extends to get out through birth canal.

So, we aimed to study the safety and feasibility of the External Pop-out (EPO) technique; a novel technique that supports the LUS during fetal head extraction with its effect on the rate of uterine incision extensions.

\section{PATIENTS AND METHODS}

The current study is a registered prospective cohort study (NCT02755168) compassing the new technique, EPO, for head extraction during CS. The ethical review board of the Faculty of Medicine of the Assiut University approved the study. The participants were recruited from our Obstetrics Outpatients Clinics of the Woman's Health Hospital, Assiut University, Egypt. It was carried out in the period between the first of February 2015 and the first of February 2016.

All pregnant women ( $>37$ weeks based on the first day of the last menstrual period), who were coming to the mentioned clinic for elective CS, were invited to participate in our study. The recruited women were entered the screening phase of the study including history taking, clinical examination and body mass index (BMI) assessment. Then, two-dimensional transabdominal US was done to assess fetal number, presentation, placental site and amniotic fluid volume. A written consent was taken after counseling and discussion of the alternative techniques. Patients who were unable to give consent were excluded.

\section{Inclusion criteria}

Women were considered eligible if they were pregnant in single, living, term ( $>37$ weeks), cephalic fetus, not in labor and finally; accepted to participate in our study.

\section{Exclusion criteria}

We excluded women with, multiple gestations, low amniotic fluid volume, premature pre-labor rupture of membranes, placenta previa and fetal congenital anomalies "e.g., hydrocephalus and intrauterine fetal death".

Initially, all patients were asked to empty their bladder before CS. All cases were operated in the theater under spinal anesthesia. The abdomen was opened through a low transverse incision and till exposure of the LUS then the uterovesical peritoneum was opened by transverse incisions including the superficial myometrium of the
LUS. The incision was deepened at the center of the LUS until opening the fetal membranes. The LUS incision was extended bluntly by moving the fingers from up-down in all cases.

EPO was performed for the selected cases as the following; after opening the LUS the obstetrician never introduce her/his hand or fingers into the LUS (Figure. 1). Alternatively, during fetal head extraction, the obstetrician puts four fingers of his right hand on the lower segment at uterovesical pouch (Figure. 2). The palmar aspect of the fingers will be resting on the uterovesical peritoneal reflection not directly on the fetal head (Figure. 3). While the palmar aspect of the fingers was facing the lower segment they were pushed deep below the fetal head.

The lower segment at that moment was supported between the fetal head from inside and obstetrician's fingers from outside. The assistant was asked to push through the abdominal wall, meanwhile, the obstetrician was guiding the head by his fingers until the head got out. Thereafter; delivery of shoulders was assisted by gentle pulling by the obstetrician and pushing of the assistant from the abdominal side. After delivering placenta and membranes the LUS incision was inspected carefully for any tears or extensions especially at its lateral angles.

The tear/extension was classified as minor when lacks all the following three criteria; tearing the uterine vessels, tears requiring exteriorization of the uterus or requiring down dissection of the bladder during its repair. The tear was considered major if required any of the three aforementioned criteria and/or blood transfusion in the absence of uterine atony.

Thereafter, the uterine incision was closed in doublelayer and the closure of other layers was continued as recommended

After the development of the EPO-technique by the first author "Ali Elsaman", the second part of the study was initiated to determine the learning ability to perform the new technique by 5 senior obstetricians' trainees (M.D. holders). The first author described the EPO-technique using illustrations and movie presentation to the senior obstetricians, then each one was assisted in an elective CS by the first author to guide him in learning the technique.

Each one of the senior obstetricians was asked to perform 10 elective cesarean sections using EPOtechnique for fetal head delivery to further assess the feasibility of the technique, the learning curve for mastering the technique and the easiness to perform.

The feasibility of the EPO-technique was assessed by the ability of obstetrician to do the procedure successfully. The learning curve of the technique was assessed by the 
ability of the obstetrician to start EPO-technique and the number of cases needed to master the technique. Mastering the technique was measured by simple scale of four levels as follow:

The $1^{\text {st }}$ level; the obstetrician was able to do EPO but feeling that the original extraction is easier. The $2^{\text {nd }}$ level; the obstetrician was able to do EPO at ease as the original extraction. The $3^{\text {rd }}$ level; the obstetrician was able to do EPO better than the original extraction. The $4^{\text {th }}$ level; the obstetrician was able to do EPO better and faster than original extraction.

The easiness score (ES) was evaluated after the initial 5 cases then after the end of the 10 cases. The ES was calculated using a graduated Likert-type scale from zero to 100; in which zero means terribly difficult and 100 means very easy. For comparison; the first 5 cases were considered (early cases) while the last 5 cases were considered (late cases).

Collected data were reviewed and analyzed using the Statistic Package for Social Science Version 21 (SPSS 21.0) for Windows. Qualitative data were expressed as frequency and percentage. Quantitative data were presented in terms of mean, median, range and standard deviation. Comparison between quantitative data of easiness score was done by Mann-Whitney U test. $P$-value $<0.05$ was considered statistically significant..

\section{RESULTS}

Three-thousands and five hundred pregnant women were examined during the study period, of them 1297 (37\%) women were admitted for elective CS and invited to participate in the study. A total of 877 women were eligible for inclusion in the study, while420 patients were excluded (Figure 4, the study flowchart).

The mean age of the participants was $28.16 \pm 6.07$ years and the mean BMI was $26.353 .6 \pm \mathrm{Kg} / \mathrm{m}^{2}$. The socio-demographic characteristics of the study participants and the different indications of cesarean sections were presented in table 1 . Repeat CS was the most common indication of CS $(53.14 \%)$. The EPOtechnique was successful in $845 / 877$ cases (96.3\%) but impossible in the remaining 32 cases because of extensive pelvic adhesions (Table 1).

As regard the incidence of extensions; minor extensions associated with EPO observed in 8/ 845 $(0.95 \%)$ while there were no reported cases of a major extension in succeeded cases. However; there were 5 cases had extensions after the failure of EPO, 2 of them had major extensions.

As regard the second part of the study; the success rate of the obstetricians' trainees to perform the EPO- technique was $96 \%$ (48/ 50 cases) which is nearly similar to the success rate of the first author (Table 2).

As regard the learning curve of the trainees for mastering the EPO-technique; all of the trainees attained the $4^{\text {th }}$ level by their sixth case, so the least number of cases needed for mastering the technique was to perform 5 cases independently (Figure. 5). These results confirmed also by the easiness scores reported by the trainees after each case. There was a statistically significant difference between the mean of ES in the early 5 cases and the next 5 cases in 4 out of 5 trainees. The remaining trainee reported a higher ES earlier from his $4^{\text {th }}$ case. The mean of total scores in the late cases was also significantly higher than the early cases $(p=0.0001)$ (Table 2$)$.

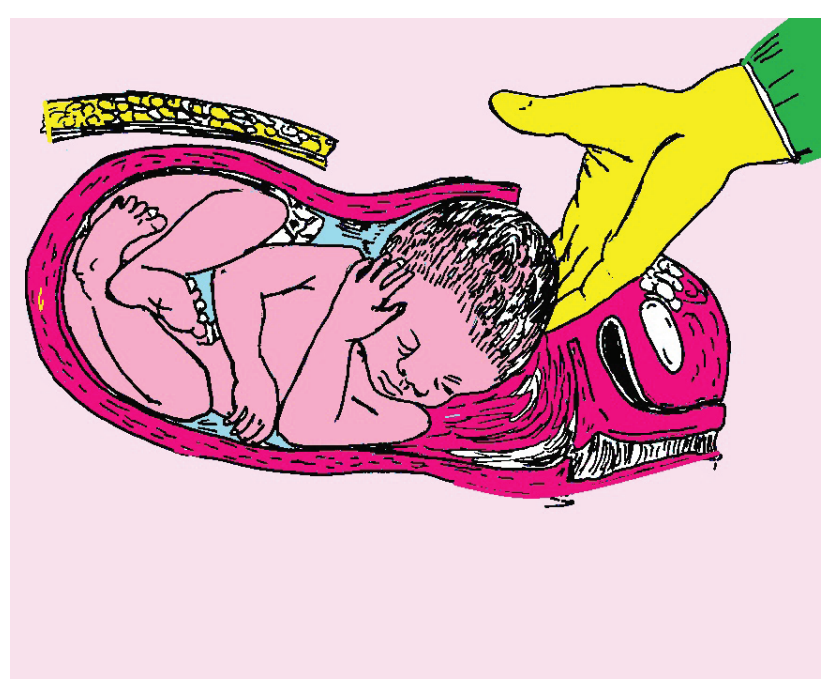

Figure. 1: Classic fetal head extraction After opening the lower segment, the obstetrician introduces his hand into the lower uterine segment.

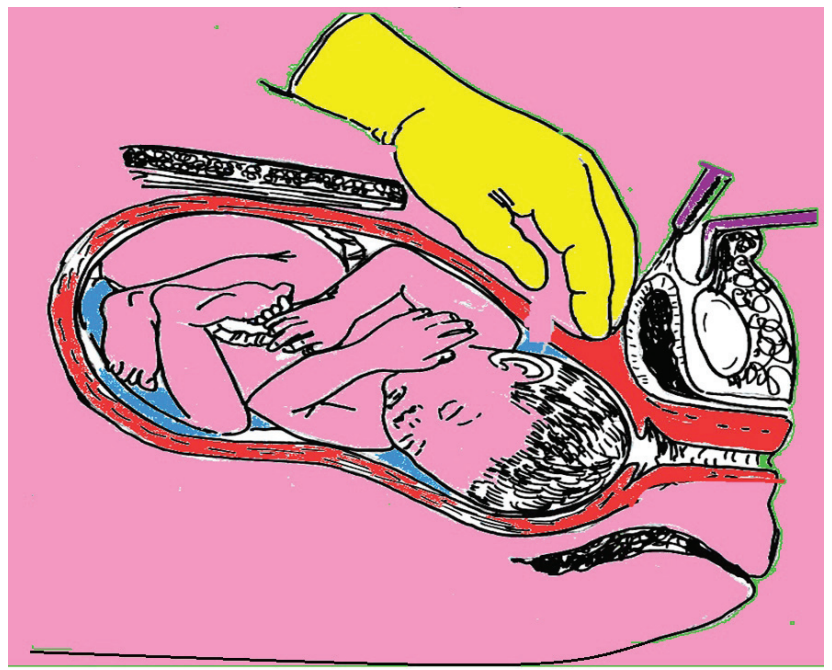

Figure. 2: External Pop-out Technique The initial direction of the fingers during fetal head extraction as the obstetrician puts four fingers of his right hand on the lower segment at uterovesical pouch. 


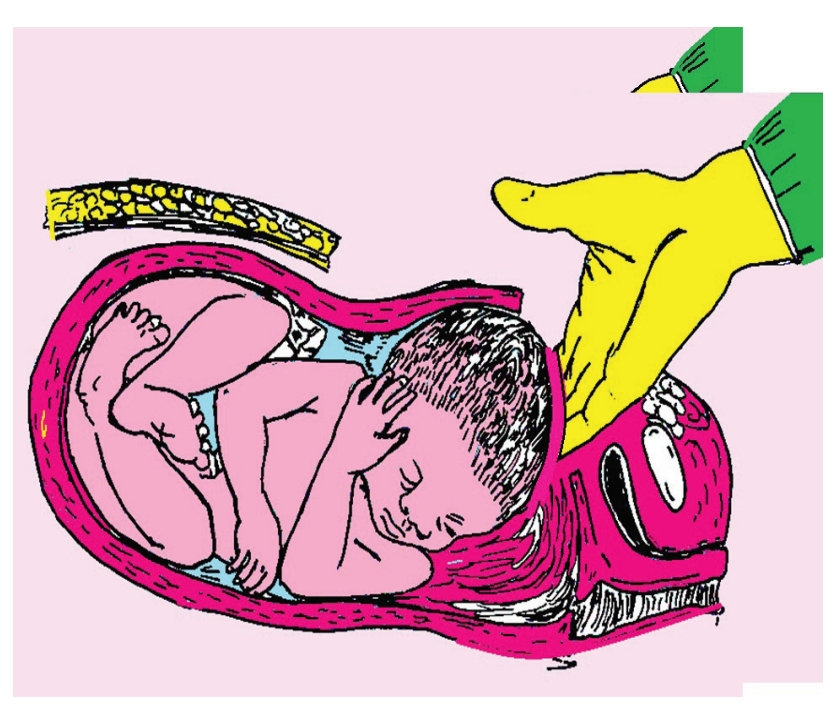

Figure 3: External Pop-out Technique The palmar aspects of the fingers are resting on the uterovesical peritoneal reflection not directly on the fetal head. So, the lower uterine segment is supported between the head and the palm of the obstetrician.

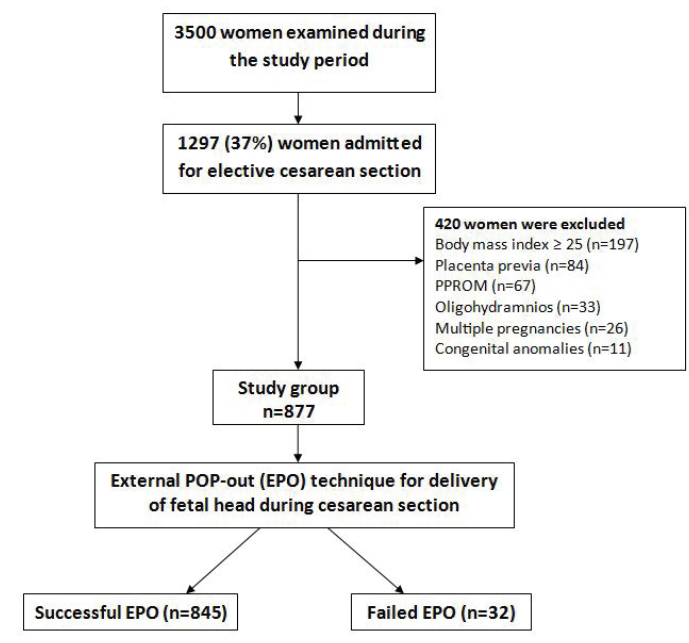

Figure 4: The study flowchart

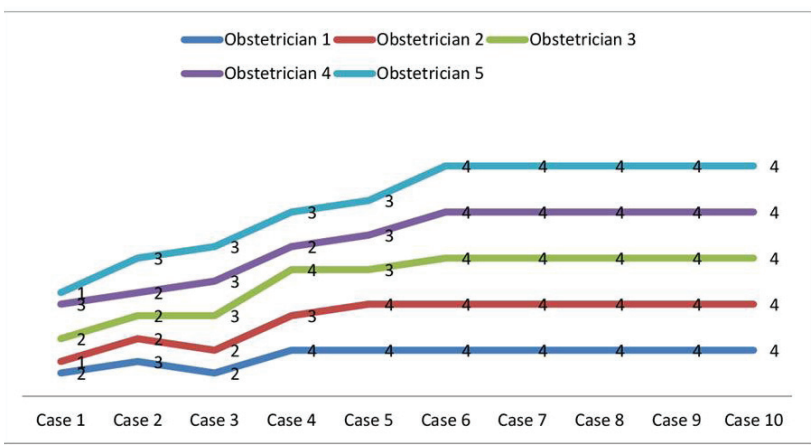

Figure 5: The learning curve of the senior obstetricians' trainee in 10 cases.

Table 1: The study outcomes

\begin{tabular}{lc}
\hline Variables & $\begin{array}{c}\text { Study participants } \\
(\mathbf{n}=\mathbf{8 7 7})\end{array}$ \\
\hline Age (years), mean \pm SD & $28.17 \pm 6.07$ \\
\hline BMI (Kg/m2), mean \pm SD & $26.35 \pm 3.6$ \\
\hline Parity, mean \pm SD & $2.47 \pm 1.61$ \\
\hline previous CS, median (range) & $2(0-5)$ \\
\hline Previous miscarriage, median (range) & $1(0-4)$ \\
\hline $\begin{array}{l}\text { Gestational age at delivery (weeks), } \\
\text { mean } \pm \text { SD }\end{array}$ & $38.27 \pm 1.39$ \\
\hline Indications of CS: $\mathbf{n}$ (\%) & $466(53.14 \%)$ \\
Repeat CS & $292(33.29 \%)$ \\
CS on request & $76(8.67 \%)$ \\
Malpresentation & $43(4.9 \%)$ \\
\hline Cephalopelvic disproportion & BMI; body mass index, CS; cesarean section, SD; standard deviation
\end{tabular}

Table 2: The success rate and easiness score (ES) of performance for EPO-technique by senior obstetricians' trainees

\begin{tabular}{lcccc}
\hline Trainees & $\begin{array}{c}\text { Success rate } \\
(\mathbf{n}=\mathbf{1 0}) \boldsymbol{n}(\%)\end{array}$ & $\begin{array}{c}\text { ES for the early cases } \\
\text { Mean } \pm S D\end{array}$ & $\begin{array}{c}\text { ES for the late cases } \\
\text { Mean } \pm S D\end{array}$ & $\boldsymbol{P}_{\text {-value }}$ \\
\hline Obstetrician 1 & $10(100 \%)$ & $78 \pm 20.18$ & $96.6 \pm 3.22$ & 0.171 \\
\hline Obstetrician 2 & $9(90 \%)$ & $61 \pm 24.85$ & $97 \pm 2.74$ & $\mathbf{0 . 0 3 1}^{\text {a }}$ \\
\hline Obstetrician 3 & $10(100 \%)$ & $62 \pm 13.96$ & $97 \pm 2.83$ & $\mathbf{0 . 0 1 6}^{\text {a }}$ \\
\hline Obstetrician 4 & $10(100 \%)$ & $66 \pm 18.51$ & $95 \pm 4.39$ & $\mathbf{0 . 0 0 8}^{\text {a }}$ \\
\hline Obstetrician 5 & $9(90 \%)$ & $61 \pm 23.29$ & $96.24 \pm 3.69$ & $\mathbf{0 . 0 0 8}^{\text {a }}$ \\
\hline Total & $48 / 50(96 \%)$ & $65.6 \pm 19.86$ & $\mathbf{0 . 0 0 0 1}^{\text {a }}$ \\
\hline
\end{tabular}

ES; easiness score, SD; standard deviation, ( $\left(^{a}\right)$ statistical significant difference

Comparison between easiness scores was done by Mann-Whitney U test 


\section{DISCUSSION}

Cesarean section is the most common surgical procedure, practiced on a daily basis all over the world with continuous rising rates, so it is being the subject of ceaseless refinement to avoid any complications. In the current study, we introduced a novel procedure for fetal head extraction during CS through supporting the lower uterine segment to minimize the rate of unintended extensions of CS incision. The External Pop-out technique is safe, effective and feasible for fetal head extraction during elective CS.

Delivery of the fetal head through the uterine incision is one of the major technical problems during elective $\mathrm{CS}$, especially when the presenting part is non-engaged. All of the previously described procedures, alternatives to the classic manual head extraction, were inconclusive and not convincing to the obstetricians for routine use. Also, no reported well-designed trials favor one of them over other. Application of vacuum cup, use of forceps blade, increasing fundal pressure and making additional uterine incisions were previously reported as alternative techniques. None of them gain any popularity or proved to be used as a basic step during CS, besides many complications were reported in mothers and infants ${ }^{8-11}$.

In the present study, the EPO-technique was performed successfully in $845 / 877$ cases (96.3\%), this refers to the feasibility and reproducibility of performing EPO-technique that assumed to minimize operative complications of CS. However; the procedure was not possible in the remaining 31 cases because of extensive pelvic adhesions that were completely obliterating the uterovesical pouch after previous pelvic surgeries and one case due to the presence of myoma occupying the lower segment.

The initial results of the current research revealed that EPO-technique was associated with low chance of incision extension whereas only $8 / 845(0.94 \%)$ minor extensions that repaired easily without downward dissection of the bladder, exteriorization of the uterus or blood transfusion. The present rate of LUS incision extensions is 4 to 16 folds less that reported with the conventional methods of fetal head extraction ${ }^{2,3}$.

On the other hand, minor extensions occurred at a much higher rate $3 / 32(9.7 \%)$ in those with failed EPO-technique. This represents a 10 fold increase in the rate of LUS incision extensions. In addition, 2 other cases $(6.25 \%)$ had major extensions as both required blood transfusion, dissection of the bladder due to an involvement of the uterine vessels and uterine exteriorization which was not possible in one case because of uterine fixity. However, comparing cases with no or minimal adhesions with cases with extensive adhesions seems to be biased and actual difference should be obtained from randomized trials comparing like with like.

Nevertheless, the reported unintended extension rate in cases with failed EPO is higher than the reported rate $(4.8 \%)$ by Asicioglu et al, when they used blunt extension technique ${ }^{2}$. This difference could be explained by the nature of cases in which EPO-technique failed; all were having adhesions and fixity of the LUS. This represents a weakness point in this comparison; however, the major objective of our study was not to compare but rather to study the feasibility, easiness and learning curve.

In spite of the tremendous increase in the rates of CS, the technique of delivery of fetal head has undergone very little modifications. In 1984, Pelosi and Apuzzio successfully used the silicone obstetric vacuum cup for delivery of fetal head during $\mathrm{CS}^{12}$. They considered it as an effective and harmless method alternative to the classic method of head extraction. Although this approach may reduce the risk of unintended uterine incision extensions, only one RCT reported compared its safety and feasibility with the classic manual head extraction ${ }^{11}$.

Moreover, Sritippayawan and Chantrapitak recommended the use of vacuum extractor during CS, as it was a non-traumatic and rapid method but associated with more blood loss ${ }^{11}$. Noteworthy, they didn't report the rate of uterine incision extensions or occurrence of other complications during CS. We think that using more instruments during CS will be technically difficult for young obstetricians, time-consuming and adding more costs for re-sterilization of the vacuum cups and tubes with every cesarean delivery, unlike our new described technique.

The current technique is considered a modification in the classic method of fetal head delivery to keep the procedure easy, simple, and also less traumatic to the uterus through support the LUS while extracting the fetal head. This avoids insertion of the obstetrician's hand inside the uterine cavity which definitely exerts more stretch on the LUS and increases its vulnerability to tear or incision extensions.

Our results were satisfactory regarding the participants, and no complications were reported apart from the 8 cases of minor extensions. These figures support the efficacy and safety of our procedure in comparison with the classic options. However, larger comparative studies are still required to give a clear recommendation for such cases. The authors are now working on a large randomized controlled study for that purpose. 


\section{CONCLUSION}

External Pop-out (EPO) technique is feasible with low rates of uterine incisions extensions. The procedure seems to be comparable in safety to the classic method with a lower rate of intraoperative complications. It carries the advantage of being simple, easy, with a short learning curve. This encourages the use of the technique for many obstetricians.

\section{CONFLICT OF INTEREST}

The Authors report no conflict of interest

\section{REFERENCES}

1. Betrán AP, Ye J, Moller AB, Zhang J, Gülmezoglu $\mathrm{AM}$, Torloni MR. The Increasing Trend in Caesarean Section Rates: Global, Regional and National Estimates: 1990- 2014. PLoS One 2016;11(2): e0148343.

2. Asicioglu O, Gungorduk K, Asicioglu BB, Yıldırım G, Gungorduk OC, Ark C. Unintended extension of the lower segment uterine incision at cesarean delivery: A randomized comparison of sharp versus blunt techniques. Am J Perinatol 2014;31(10): 837 -844.

3. Xu LL, Chau AM, Zuschmann A. Blunt vs. sharp uterine expansion at lower segment cesarean section delivery: a systematic review with metaanalysis. Am J Obstet Gynecol 2013; 208(1): 62.e162-.e18.

4. Dahlke JD, Mendez-Figueroa H, Rouse DJ, Berghella V, Baxter JK, Chauhan SP. Evidence-based surgery for cesarean delivery: An updated systematic review. Am J Obstet Gynecol 2013; 209(4): 294 -306.
5. Encarnacion B, Zlatnik MG. Cesarean delivery technique: Evidence or tradition? A review of the evidence-based cesarean delivery. Obstet Gynecol Surv 2012; 67(8): 483- 494.

6. Walsh CA. Evidence-based cesarean technique. Curr Opin Obstet Gynecol 2010; 22(2): 110- 115.

7. Berghella V, Baxter JK, Chauhan SP. Evidence-based surgery for cesarean delivery. Am J Obstet Gynecol 2005; 193(5): 1607- 1617.

8. Clark SL, Vines VL, Belfort MA. Fetal injury associated with routine vacuum use during cesarean delivery. Am J Obstet Gynecol 2008; 198(4): e4.

9. Kim TY, Ryu DH. The effect of fundal pressure at Caesarean section on maternal haemodynamics. Anaesthesia 2006; 61(5): 434- 438.

10. Nakano R. Use of the vacuum extractor for delivery of the fetal head at cesarean section. Am J Obstet Gynecol 1981; 141(4): 475- 476.

11. Sritippayawan S, Chantrapitak W. Assisted delivery of high floating fetal head: a comparison of vacuumassisted delivery with manual extraction. Asian Biomed 2011; 5: 699 -703.

12. Pelosi MA, Apuzzio J. Use of the soft, silicone obstetric vacuum cup for delivery of the fetal head at cesarean section. J Reprod Med 1984;29(4): $289-292$. 